\title{
BMJ An observational study of the hand open hygiene initiative: a comparison of preintervention and postintervention outcomes
}

\author{
Amit Mukerji, ${ }_{1}$ Janet Narciso, ${ }^{2}$ Christine Moore, ${ }^{3}$ Allison McGeer, ${ }^{4}$ Edmond Kelly, ${ }^{1}$ \\ Vibhuti Shah $^{1}$
}

To cite: Mukerji A, Narciso J, Moore C, et al. An observational study of the hand hygiene initiative: a comparison of preintervention and postintervention outcomes. BMJ Open 2013;3:e003018. doi:10.1136/bmjopen-2013003018

- Prepublication history for this paper are available online. To view these files please visit the journal online (http://dx.doi.org/10.1136/ bmjopen-2013-003018).

Received 9 April 2013 Revised 20 April 2013 Accepted 23 April 2013

This final article is available for use under the terms of the Creative Commons Attribution Non-Commercial 2.0 Licence; see http://bmjopen.bmj.com

For numbered affiliations see end of article.

Correspondence to Dr Vibhuti Shah; vshah@mtsinai.on.ca

\section{ABSTRACT}

Objectives: To evaluate the impact of implementing a simple, user-friendly eLearning module on hand hygiene $(\mathrm{HH})$ compliance and infection rates.

Design: Preintervention and postintervention observational study.

Participants: All neonates admitted to the neonatal intensive care unit (NICU) over the study period were eligible for participation and were included in the analyses. A total of 3422 patients were admitted over a 36-month span (July 2009 to June 2012).

Interventions: In the preintervention and postintervention periods (phases I and II), all healthcare providers were trained on $\mathrm{HH}$ practices using an eLearning module. The principles of the ' 4 moments of $\mathrm{HH}$ ' and definition of 'baby space' were incorporated using interactive tools. The intervention then extended into a long-term sustainability programme (phase III), including the requirement of an annual recertification of the module and introduction of posters and screensavers throughout the NICU.

Primary and secondary outcome measures: The primary outcome was $\mathrm{HH}$ compliance rates among healthcare providers in the three phases. The secondary outcome was healthcare-associated infection rates in the NICU.

Results: HH compliance rates declined initially in phase II then improved in phase III with the addition of a longterm sustainability programme $(76 \%, 67 \%$ and $76 \%$ in phases I, II and III, respectively $(p<0.01)$. Infection rates showed an opposing, but concomitant trend in the overall population as well as in infants $<1500 \mathrm{~g}$ and were $4 \%, 6 \%$ and $4 \%(p=0.02)$, and $11 \%, 21 \%$ and $16 \%$ $(p<0.01)$, respectively, during the three phases.

Conclusions: Interventions to improve $\mathrm{HH}$ compliance are challenging to implement and sustain with the need for ongoing reinforcement and education.

\section{INTRODUCTION}

Healthcare-associated infections (HAIs), defined as infections acquired during therapy for another condition and not

\section{ARTICLE SUMMARY}

Article focus

- Non-compliance with hand hygiene $(\mathrm{HH})$ practices is linked with healthcare-associated infections, a major cause of mortality and morbidity.

- Can implementation of a simple eLearning module followed by a reinforcement programme be associated with a sustained increase in $\mathrm{HH}$ compliance in a neonatal intensive care unit (NICU)?

Key messages

- An intervention using a simple eLearning module coupled with a reinforcement programme did not lead to sustained improvement in $\mathrm{HH}$ compliance.

- It is difficult to change practice among healthcare providers with a single educational intervention, particularly in a busy intensive care setting.

- Future work needs to utilise creative approaches to increase $\mathrm{HH}$ compliance and maintain sustainability.

Strengths and limitations of this study

- The strengths of this study include a large sample size in a tertiary level 3 NICU, the inclusion of both $\mathrm{HH}$ compliance and healthcare-associated infections as outcomes and the implementation of a reinforcement programme to maintain longterm sustainability.

- The weaknesses include the lack of a control group and the use of HH audits over 3 months as a surrogate for compliance rates for the entire year.

present or incubating at the time of admission, comprise a significant burden of illness. Up to $5-10 \%$ of hospitalised patients in industrialised nations are affected ${ }^{1}$ with even higher rates in the neonatal intensive care unit (NICU). For example, in Europe an incidence of $25.6 \%$ was noted in NICUs over a 6 -year period. ${ }^{2}$ In the USA, using a point prevalence survey design, 116 HAIs were 
documented from 827 admissions across 29 NICUs (14/ 100 admissions). ${ }^{3}$ Most of these cases occurred in low birth weight $(\mathrm{BW})$ neonates with a relative risk of 11.8 (95\% (CI) 7.6 to 18.18 ) in infants $<1000 \mathrm{~g} .{ }^{4}$ They have a significant economic impact with one study estimating an annual cost of $\$ 100$ million dollars for treatment of HAIs in infants $<1500 \mathrm{~g}$ in the USA alone. ${ }^{5}$

Risk factors linked with HAIs in NICUs include the use of indwelling vascular catheters, assisted ventilation and prolonged hospital stays in subjects with a relatively compromised immune system. ${ }^{6}$ However, hand contamination is a significant underlying problem and proper hand hygiene $(\mathrm{HH})$ has been repeatedly demonstrated to be the single most important infection control activity in the NICU. ${ }^{7-10}$ In fact, $\mathrm{HH}$ has been incorporated as a core component of the Global Patient Safety Challenge initiative 'Clean Care is Safer Care', set up by the WHO in 2005 with the goal of reducing the burden of HAIs worldwide. ${ }^{11}$ Despite recognising that $\mathrm{HH}$ is crucial in reducing infection rates, compliance rates of $\mathrm{HH}$ among healthcare providers (HCPs) remain low. In a recent systematic review of 96 studies (with 65 studies in intensive care settings) on $\mathrm{HH}$ compliance of HCPs from industrialised nations, it was noted that compliance rates were as low as $30-40 \%$ in intensive care settings compared with $50-60 \%$ in other settings. ${ }^{12}$

Given the importance of the need to improve $\mathrm{HH}$ compliance among HCPs, the Ministry of Health and Long Term Care (MOHLTC) in the province of Ontario, Canada, initiated a programme entitled 'Just Clean Your Hands' in 2008. The programme was based on the 'Clean Care is Safer Care' WHO initiative and provides a step-by-step guide on how to implement strategies to improve and sustain compliance. ${ }^{13}$ Implementation of multifaceted approaches similar to 'Just Clean Your Hands' to improve HH compliance at institutional levels (including the use of simple education initiatives (posters, screen savers), simplification of the hand-washing process using alcohol-based rubs and involving HCPs in decision-making) has been met with varying degrees of success. ${ }^{14}$ 15 In fact, a Cochrane review evaluating the success of implementation of interventions to improve $\mathrm{HH}$ compliance among HCPs concluded that multifaceted campaign approaches seem to have an effect, but firm conclusions could not be drawn. ${ }^{16}$

The NICU at Mount Sinai Hospital in Toronto, Canada, is among the largest in North America, with approximately 1100 admissions annually with approximately $30 \%$ of these being neonates $<1500 \mathrm{~g}$. An assessment of $\mathrm{HH}$ compliance rates among HCPs in the Mount Sinai Hospital NICU ranged from $67 \%$ to $82 \%$ prior to the initiation of the study. With the goal of improving on such statistics, a customised HH promotion programme based on the MOHTLC's guidelines was implemented in our NICU. The purpose of this observational study was to evaluate whether this programme could be associated with an improvement on HCPs' HH compliance and HAI rates.

\section{MATERIALS AND METHODS}

This was a preintevention and postintervention observational study conducted in the levels 3 and 2 NICUs of Mount Sinai Hospital, Toronto, Canada. The data collection points spanned from 1 July 2009 to 30 June 2012. Figure 1 delineates the different phases of the study with the various interventions and $\mathrm{HH}$ audits.

\section{Study setting}

Levels 3 and 2 NICUs in Mount Sinai Hospital, Toronto, are open concept with a maximum capacity of 34 and

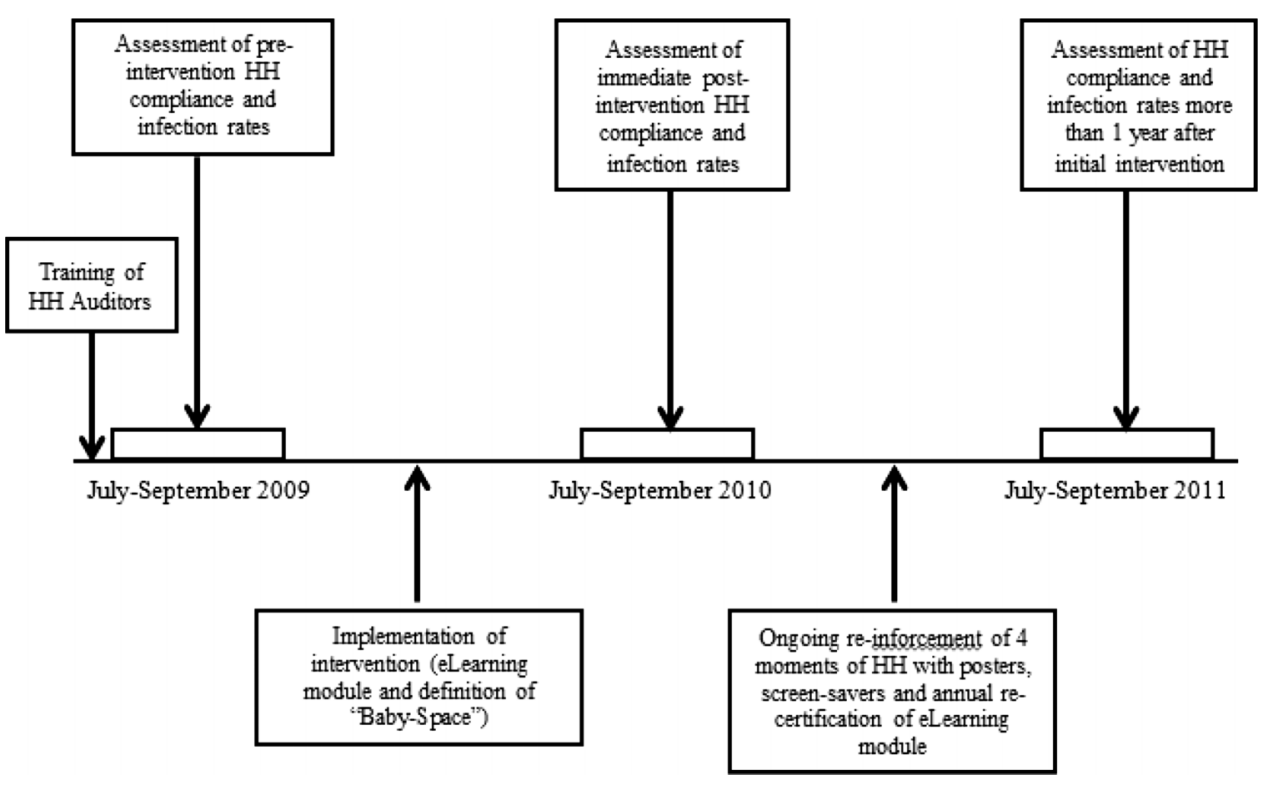

Figure 1 Timeline delineating the interventions and $\mathrm{HH}$ audits over the study period. 
20 beds in the levels 3 and 2 units, respectively. There are seven and six hand washing sinks in the level 3 and 2 units, respectively, with antiseptic hand soap, water and paper-towels available at each station. In addition, antiseptic hand rub is available at each bedside.

There are three entrances to the level 3 NICU: (1) a main entrance that has two doors with signs to wash hands before entering the unit (closed at all times with three hand washing sinks available); (2) a back entrance with access to the delivery suites (restricted to staff only) and (3) a side entrance that leads to the maternal-fetal medicine inpatient unit. Level 2 NICU has two entrances: one main entrance for families and staff and another back entrance restricted to staff.

\section{Healthcare providers}

Levels 3 and 2 NICUs at Mount Sinai Hospital currently employ a total of 171 registered nurses with 21-26 nurses working in the unit at any one point (including one charge nurse and one resuscitation nurse), with similar numbers over the last 4 years. There are 12 registered respiratory therapists employed currently, of whom 3-4 are present within the unit at any one time with an additional respiratory therapist assigned to attend deliveries. There are currently 15 faculty members on staff (up from 11 in 2009) with 2 faculty members on service on any given day in the level 3 unit and one faculty member in the level 2 unit. There are also nurse practitioners (1-2 on service at any one time) and trainees: 4-6 fellows on service at a given time (up from 2 to 3 in June 2009), residents (3-4 on service at a time) and occasionally, medical students on electives. Other HCPs include dietitians, pharmacists, social workers, a parent resource nurse and occasionally their respective trainees. There are also research assistants/data collectors working in the unit at various points, although they have minimal contact with the patients.

\section{Training of auditors}

In June 2009, volunteer students were recruited as $\mathrm{HH}$ auditors after a detailed training process. The training consisted of reviewing an interactive $\mathrm{HH}$ module created by the MOHLTC as part of the 'Just Clean Your Hands' initiative (http://www.oahpp.ca/services/jcyh), reviewing various local $\mathrm{HH}$ policies at Mount Sinai Hospital and going through detailed presentations on observation and auditing. This provided the trainees with adequate knowledge of when $\mathrm{HH}$ is required in a clinical setting, defined as an opportunity or indication, what appropriate $\mathrm{HH}$ consists of and how to complete an assessment form.

The training was followed by viewing a DVD module consisting of mock scenarios with the trainees assessing for opportunities and indications for $\mathrm{HH}$ and rating the compliance of HCPs with $\mathrm{HH}$. Then, the trainees were described the actual scenarios from the NICU with realtime discussions on the indications and opportunities. All of these were followed by trainees going into the clinical environment accompanied by at least another observer and assessing for $\mathrm{HH}$ compliance with discussions on disagreements. Once a $>95 \%$ rate of agreement was consistently reached with other observers, the trainees were deemed qualified to be an independent $\mathrm{HH}$ auditor.

\section{Phase I (preintervention: July 2009-June 2010)}

Hand hygiene compliance rates in the levels 3 and 2 NICUs were determined for the four 'moments' of $\mathrm{HH}$ as delineated by the 'Just Clean Your Hands' campaign by trained assessors. These moments were before initial patient/patient environment contact, before aseptic procedure, after body fluid exposure risk and after patient/ patient environment contact.

\section{Phase II (immediate postintervention: July 2010-June 2011)}

In December of 2009, an eLearning module was created and implemented to train HCPs on the four moments of $\mathrm{HH}$ as described above (https://tahsn.pathlore.net/ tahsn/courseware/MSH/NICU\%20hand\%20hygiene/ player.html). This module consisted of education material that included a detailed description of the moments of $\mathrm{HH}$ and interactive tools to further clarify what constitutes the patient environment ('baby space'), along with case scenarios. A quiz had to be completed, in which there were 10 questions with a pass mark of $80 \%$. All HCPs working in the NICUs at the time of introduction of this module completed the training by March 2010 . Subsequently, all new trainees and HCPs hired were asked to complete the module on an on-going basis. Furthermore, all HCPs were mandated to complete the eLearning module annually as a refresher. Between July and September 2010, trained HH auditors evaluated the HH compliance rates.

\section{Phase III (postintervention and retention: July 2011-June} 2012)

Following the introduction of the $\mathrm{HH}$ module, numerous posters and screensavers detailing the four moments of $\mathrm{HH}$ and baby-space were placed in the units to act as reinforcement for the need of ongoing adherence to the principles outlined in the eLearning module. This was in addition to the aforementioned annual recertification, as well as ongoing training of new employees. $\mathrm{HH}$ audits were completed again between July and September, 2011 as a representation of phase III compliance rates.

\section{Outcomes}

The primary outcome of this study was a comparison of the HH compliance rates among HCPs during the three phases as described above. The secondary outcome was the comparison of annual rates of HAIs shown to be linked with $\mathrm{HH}^{10}{ }^{10}$ Hospital-acquired infections were defined in this study as positive blood and/or cerebrospinal fluid culture noted after 2 days of age. Urinary 
tract infections (UTIs) were not included in our database of infections. The HAI rates between July and September of the 3 years were compared, corresponding directly with the periods of $\mathrm{HH}$ auditing. Using the Mount Sinai Hospital Infection Control Programme database, which contains information from all positive culture results obtained in the hospital, the infection rates for the NICU were determined for all patients admitted, stratified by BW and gestational age (GA), and compared for the periods before and after the intervention.

\section{Statistical analyses}

Demographic characteristics, compliance and HAI rates between the three phases and neonatal outcomes between the groups were compared using $\chi^{2}$ or Fisher's exact test for categorical data and analysis of variance for continuous data as appropriate (SAS institute, Cary, North Carolina, USA). A $p$ value of $<0.05$ was considered significant.

\section{RESULTS}

\section{Patient characteristics}

All neonates admitted to the unit over the study period were eligible for participation and were included in the analyses. This amounted to 3422 patients admitted over a 36-month span (July, 2009 - June, 2012). Of these, 2059 were low BW $(<2500 \mathrm{~g} ; 60 \%$ of total admissions) and 2187 preterm ( $<37$ weeks GA; $64 \%$ of total admissions). The mean \pm standard deviation $\mathrm{BW}$ and GA at admission were $2264 \pm 1016 \mathrm{~g}$ and $33.9 \pm 4.6$ weeks, respectively. Table 1 compares the baseline patient characteristics over the course of the study. There was a statistically significant difference in the study population in phase II when compared with phases I and III. Infants in phase II were of younger GA, had lower BW with higher proportions of preterm, low birth weight and very low birth weight infants $(<1500$ grams $)$ born during that time period $(p<0.05$ for all $)$. However, the mean length of stay and days on continuous positive airway pressure support were longer for infants in phase $I(p<0.05)$.

\section{HH compliance}

The results of the HH compliance rates for all HCPs in the levels 3 and level 2 NICUs are shown in table 2.

There was a statistically significant decline during phase II for the encounters of 'before patient contact' and 'after body fluid contact' and the overall $\mathrm{HH}$ compliance despite implementation of the educational module $(p<0.05)$. However, following the reinforcement phase, the rates improved in all four domains, but the overall $\mathrm{HH}$ compliance preimplementation and postimplementation remained the same.

\section{Hospital-acquired infection rates}

The annual rates of HAIs had a trend inverse to that of $\mathrm{HH}$ compliance rates. Assessing the overall rates for all infants, as well as specifically for very low BW or preterm infants, the infection rates were statistically significantly higher when the $\mathrm{HH}$ compliance rates were low, then decreased as the $\mathrm{HH}$ compliance rates improved $(p<0.05)$. The HAI rates for the three phases of the study are shown in table 3 .

\section{DISCUSSION}

In this study, the implementation of an extensive educational intervention detailing the ' 4 moments of $\mathrm{HH}^{\mathrm{H}}$ ' and introducing the concept of 'baby space' was associated with an initial reduction in $\mathrm{HH}$ compliance rates and an increase in HAI rates. These findings were contradictory to our hypothesis that the implementation of the educational intervention would lead to improved compliance and a reduction in HAIs. It was only after ongoing reinforcement with annual recertification, introduction of posters and screensavers throughout the NICU and detailed description of what constituted 'baby-space' that the rates of $\mathrm{HH}$ compliance improved in all 4 moments of $\mathrm{HH}$ evaluated from phase II to phase III. This underscores the notion that in order to achieve long-term sustainability in compliance rates, reinforcement is needed. To our knowledge, this study is the first to evaluate the issue of compliance sustainability in an NICU setting.

\section{Table 1 Characteristics of study population}

\begin{tabular}{|c|c|c|c|c|}
\hline Variables & Phase I & Phase II & Phase III & p Value \\
\hline Number of patients & 1077 & 1148 & 1197 & \\
\hline Gestational age (weeks) (mean, SD) & $33.9(4.5)$ & $33.6(4.6)$ & $34.3(4.7)$ & $<0.01$ \\
\hline Preterm infants $<37$ weeks (n, \%) & $706(66)$ & $773(67)$ & $708(59)$ & $<0.01$ \\
\hline Birth weight (g) (mean, SD) & 2237 (1007) & $2183(993)$ & 2366 (1039) & $<0.01$ \\
\hline Low birth weight infants ( $\mathrm{n}, \%)$ & $657(61)$ & 735 (64) & $667(56)$ & $<0.01$ \\
\hline Very low birth weight infants ( $n, \%)$ & $305(28)$ & $321(28)$ & $285(24)$ & 0.02 \\
\hline Extremely low birth weight infants (n, \%) & $121(11)$ & $157(14)$ & $142(12)$ & 0.19 \\
\hline Average length of stay* (days) (mean, SD) & $18.5(26.9)$ & $16.4(24.8)$ & $14.7(24.2)$ & $<0.01$ \\
\hline Need for invasive and non-invasive ventilation (days) (mean, SD) & $2.5(8.2)$ & $2.2(7.2)$ & $2.5(9.1)$ & 0.60 \\
\hline Number of patient days CPAP (mean, SD) & $6.5(14.5)$ & $5.5(13.2)$ & $3.6(9.0)$ & $<0.01$ \\
\hline
\end{tabular}


Table 2 Hand hygiene compliance rates

\begin{tabular}{|c|c|c|c|c|}
\hline Encounters* & Phase I & Phase II & Phase III & p Value \\
\hline Before patient contact & $199 / 242(82 \%)$ & $140 / 200(70 \%)$ & $76 / 104(73 \%)$ & 0.01 \\
\hline Before aseptic procedure & $6 / 9(67 \%)$ & $11 / 20(55 \%)$ & $8 / 8(100 \%)$ & 0.18 \\
\hline After body fluid contact & $28 / 34(82 \%)$ & $19 / 38(50 \%)$ & $15 / 19(80 \%)$ & 0.01 \\
\hline After patient contact & $155 / 224(69 \%)$ & $137 / 199(69 \%)$ & $66 / 87(76 \%)$ & 0.64 \\
\hline Average hand hygiene compliance & $388 / 509(76 \%)$ & $307 / 457(67 \%)$ & $165 / 218(76 \%)$ & $<0.01$ \\
\hline
\end{tabular}

There are several possible reasons for the failure to improve $\mathrm{HH}$ compliance in phase II. The most important being the fact that HCPs had to have a good understanding of what constituted 'baby space' and apply the principles in practice, which was a major challenge in the open-concept space, particularly in a busy unit. Despite having been included in the training module, IV bags and the cover on top of the baby's isolette could have easily been not thought of as being part of 'baby space'. It was difficult to explain why the $\mathrm{HH}$ compliance rate before patient contact fell after implementation of the educational intervention. One explanation may be owing to the need for sudden and quick clinical interventions if an infant had a significant desaturation or apneic episode, which may have occurred more frequently as there were smaller and more preterm babies in phase II. Furthermore, the presence of more high-risk patients in phase II might explain the higher rates of HAIs. Another explanation for the decrease in $\mathrm{HH}$ rates may be that HCPs were extravigilant when the assessors were monitoring $\mathrm{HH}$ compliance in phase $\mathrm{I}$ (the so-called Hawthorne effect), but may have become more accustomed to their presence in phases II and III. Therefore, HH compliance rates in phase I may have been falsely misrepresentative of the actual rate.

It is well known that the widespread acceptance to a new practice takes time. It has been described by sociologist Everett Rogers as part of the Diffusion of Innovations theory, ${ }^{17}$ which notes that acceptance and adoption of an innovation happens in stages whereby some people are 'early adopters', others constitute the 'early majority', while many others are in the 'late majority'. It is only after reaching a critical mass that a change in practice is able to self-sustain, hence the need for ongoing reinforcement. Moreover, change can only happen if the practice is consistent with habits, values and experiences of the potential adopters and that it provides tangible results. In the context of $\mathrm{HH}$, despite the described efforts, the behaviour of HCPs is difficult to modify and that quarterly audits may have not been sufficient to alter their behaviour.

The difficulty in changing practice in medicine after a single intervention is not a new concept. Published studies in both NICUs and other settings have yielded mixed results despite the use of multifaceted approaches. ${ }^{14-16}$ Pittet et al showed that a hospital-wide campaign including posters and performance feedback led to an increase in $\mathrm{HH}$ compliance rates from $48 \%$ to $66 \%(\mathrm{p}<0.001)$ with a concomitant decrease in nosocomial infection (NI) rates from $16.9 \%$ to $9.9 \%$ $(\mathrm{p}=0.04))^{18}$ Huang et al performed a randomised controlled trial of 100 nurses, with the study group receiving an educational intervention consisting of a $2 \mathrm{~h}$ lecture on blood-borne pathogens and universal precautions and a $1 \mathrm{~h}$ demonstration of universal precaution techniques. $^{14}$ An observation period of $30 \mathrm{~min}$ noting the number of instances of $\mathrm{HH}$ showed higher compliance 4 months postintervention, indicating sustained benefits of this intervention. On the other hand, an earlier study by Gould et al failed to show any effect of an educational campaign on $\mathrm{HH}$ rates. ${ }^{19}$ Another study evaluating the impact of a single-intervention promotion programme on $\mathrm{HH}$ practices in an NICU setting showed a dramatic increase in $\mathrm{HH}$ compliance from $6.3 \%$ to $81.2 \%$ 7 months after the programme $(\mathrm{p}<0.001)$. However, there was no decrease in the infection rates. ${ }^{20}$ Helder et $a l^{21}$ studied the effectiveness of a $\mathrm{HH}$ education programme on the incidence of NI in an urban NICU. Hand hygiene compliance increased before patient contact $(88 \%$ vs $65 \%, \mathrm{p}<0.001)$ with a statistically significant decrease in infection rates. However, this was a single intervention with no provision for ongoing training and long-term sustainability. Whether such an intervention results in long-lasting effects beyond the study period remains unknown.

Our study has several strengths. These include (1) inclusion of $\mathrm{HH}$ compliance and HAI rates as outcome measures; (2) implementation of measures

Table 3 Hospital acquired infection rates

\begin{tabular}{lllll}
\hline Rates & Phase I & Phase II & Phase III & p Value \\
\hline All infants & $40 / 1077(4 \%)$ & $70 / 1148(6 \%)$ & $52 / 1197(4 \%)$ & 0.02 \\
VLBW $(<1500 \mathrm{~g})$ & $35 / 305(11 \%)$ & $66 / 321(21 \%)$ & $45 / 285(16 \%)$ & $<0.01$ \\
Preterm (<37 weeks GA) & $38 / 706(5 \%)$ & $70 / 773(9 \%)$ & $48 / 708(7 \%)$ & 0.02 \\
\hline GA, gestational age; VLBW, very low birth weight. & &
\end{tabular}


regarding long-term sustainability; (3) direct observation of $\mathrm{HH}$ compliance as opposed to a proxy measure (such as amount of alcohol-based hand wash used); (4) differentiation of the compliance for the four moments so that deficiencies can be indentified to target and provide education regarding specific situations;and (5) no other studies were ongoing at the time of this study targeting HAIs specifically, reducing the risk of confounding the results of this study.

The major limitations of our study include (1) the inability to include a control group (such a study would be unethical to perform); (2) the potential for the occurrence of the 'Hawthorne effect', which, as described earlier, can lead to an inflated increase in the rate of $\mathrm{HH}$ compliance owing to direct observation; (3) the use of a limited audit duration (between July and September of each year) as a surrogate for compliance throughout the year and (4) the lack of inclusion of UTIs in the HAI rates.

Hand hygiene compliance has to continue to remain an important area of work in order to reduce rates of HAIs and provide patients with the best quality of care. As described above, numerous studies have reported various interventions, but with mixed results. Therefore, innovative approaches need to be considered. An emerging body of literature is evaluating the role of patients in education and patient-safety. ${ }^{22}{ }^{23}$ An analogous approach in the NICU would be to empower parents in promoting and improving $\mathrm{HH}$ compliance. Parents can monitor $\mathrm{HH}$ compliance and give real-time feedback to HCPs responsible for the care of their infant. Other approaches that deem consideration include the provision of incentives for $\mathrm{HH}$ compliance, giving real-time feedback from $\mathrm{HH}$ auditors by conducting random safety audits and addressing this issue from an organisational standpoint. This requires the creation of quality improvement teams focusing on workload demands and promoting a patient-safety culture. Such an approach needs to be supplemented with a 'buy-in' from all HCPs as well as administrators, as opposed to the dissemination of an eLearning module which may in and of itself be inadequate without a proper context. It is only with innovative approaches, supplemented with the proper motivation from all involved in patient care, that $\mathrm{HH}$ compliance and associated HAIs can be effectively addressed.

In conclusion, this study confirms the challenges of improving $\mathrm{HH}$ compliance among HCPs. Even though the 'Hand Hygiene Initiative' is a simple intervention, it is exceedingly difficult to implement and sustain. This study also adds to the body of literature showing that a single one-time intervention may only have a minimal effect and that on-going reinforcement is the key to maintaining $\mathrm{HH}$ compliance. Future studies directly comparing different educational interventions, along with innovative and creative approaches are needed to identify the best way to improve $\mathrm{HH}$ compliance rates. Furthermore, $\mathrm{HH}$ is only a part of the challenge in lowering HAIs and other infection control measures should be in place to reduce HAIs.

\section{Author affiliations}

${ }^{1}$ Department of Paediatrics, Mount Sinai Hospital, University of Toronto, Toronto, Ontario, Canada

${ }^{2}$ Department of Nursing, Mount Sinai Hospital, University of Toronto, Toronto, Ontario, Canada

${ }^{3}$ Department of Infection Control, Mount Sinai Hospital, University of Toronto, Toronto, Ontario, Canada

${ }^{4}$ Department of Microbiology, Mount Sinai Hospital, University of Toronto, Toronto, Ontario, Canada

Acknowledgements The authors would like to acknowledge Woojin Yoon for helping with data extraction and statistical analyses.

Contributors AM was responsible for the design and concept of the study as well as writing the original manuscript draft and subsequent draft edits (revisions). JN was responsible for the study design and concept and was involved in the dissemination of the eLearning module and subsequent sustainability measures described in the manuscript. CM was responsible for organising the hand hygiene compliance audits and reviewing the manuscript drafts. AM was responsible for collecting and providing data on infection rates and providing input in the manuscript drafts. EK was responsible for the design and concept of the study along with the creation and implementation of the eLearning module. VS was responsible for the design and concept of the study, involved in the design of the eLearning module and implementation, reviewing all the drafts of the manuscript and writing the final manuscript draft. All authors have read and approved the final manuscript.

Funding This research received no specific grant from any funding agency in the public, commercial or not-for-profit sectors.

\section{Competing interests None.}

Ethics approval The study was approved by the ethics review board at Mount Sinai Hospital.

Provenance and peer review Not commissioned; externally peer reviewed.

Data sharing statement No additional data are available.

\section{REFERENCES}

1. Pittet D, Allegranzi B, Storr J, et al. 'Clean Care is Safer Care': the Global Patient Safety Challenge 2005-2006. Int J Infect Dis 2006;10:419-24.

2. Molina-Cabrillana J, Santana-Reyes C, Hernandez J, et al. Incidence of nosocomial infections at a neonatal intensive care unit: a six year surveillance study. Enferm Infecc Microbiol Clin 2006;24:307-12.

3. Sohn AH, Garrett DO, Sinkowitz-Cochran RL, et al. Prevalence of nosocomial infections in neonatal intensive care unit patients: Results from the first national point-prevalence survey. $J$ Pediatr 2001;139:821-7.

4. Su BH, Hsieh HY, Chiu HY, et al. Nosocomial infection in a neonatal intensive care unit: a prospective study in Taiwan. Am J Infect Control 2007;35:190-5.

5. Payne NR, Carpenter JH, Badger GJ, et al. Marginal increase in cost and excess length of stay associated with nosocomial bloodstream infections in surviving very low birth weight infants. Pediatrics 2004;114:348-55.

6. Baltimore RS. Neonatal nosocomial infections. Semin Perinatol 1998;22:25-32.

7. Pessoa-Silva CL, Dharan S, Hugonnet S, et al. Dynamics of bacterial hand contamination during routine neonatal care. Infect Control Hosp Epidemiol 2004;25:192-7.

8. American Academy of Pediatrics and American College of Obstetricians and Gynecologists. Infection control. In: Hauth JC, Merenstein GB.eds Guidelines for perinatal care. 4th edn. Elk Grove Village, IL: American Academy of Pediatrics, 1997:251-77.

9. Won SP, Chou HC, Hsieh WS, et al. Handwashing program for the prevention of nosocomial infections in a neonatal intensive care unit. Infect Control Hosp Epidemiol 2004;25:742-6. 
10. Pessoa-Silva CL, Hugonnet S, Pfister R, et al. Reduction of health care associated infection risk in neonates by successful hand hygiene promotion. Pediatrics 2007;120:382-90.

11. Allegranzi B, Storr J, Dziekan G, et al. The First Global Safety Challenge "Clean Care is Safer Care": from launch to current progress and achievements. J Hosp Infect 2007;65(Suppl 2):115-23.

12. Erasmus V, Daha TJ, Brug $\mathrm{H}$, et al. Systematic review of studies on compliance with hand hygiene guidelines in hospital care. Infect Control Hosp Epidemiol 2010;31:283-94.

13. Ministry of Health and Long Term Care 'Just Clean Your Hands'. http://www.health.gov.on.ca/en/ms/handhygiene/pilotstudy.aspx (accessed 10 Sep 2012).

14. Huang J, Jiang D, Wang $X$, et al. Changing knowledge, behavio and practice related to universal precautions among hospital nurses in China. J Contin Educ Nurs 2002;33:217-24.

15. Whitby M, McLaws ML, Slater K, et al. Three successful interventions in health workers that improve compliance with hand hygiene: is sustained replication possible? Am J Infect Control 2008;36:349-55.

16. Gould DJ, Chudleigh JH, Moralejo D, et al. Interventions to improve hand hygiene compliance in patient care. Cochrane Database Syst Rev 2010;(9):CD005186.
17. Rogers E. Diffusions of innovations. 5th edn. New York, NY: Free Press, 2003:282-5.

18. Pittet D, Hugonnet S, Harbath S, et al. Effectiveness of a hospital-wide programme to improve compliance with hand hygiene. Lancet 2000;356:1307-12.

19. Gould DJ, Chamberlain A. The use of a ward-based educational teaching package to enhance nurses' compliance with infection control procedures. J Clin Nurs 1997;6:55-67.

20. Picheansathian W, Pearson A, Suchaxaya P. The effectiveness of a promotion programme on hand hygiene compliance and nosocomial infections in a neonatal intensive care unit. Int $J$ Nurs Pract 2008:14:315-21.

21. Helder OK, Brug J, Looman CW, et al. The impact of an education program of hand hygiene compliance and nosocomial infection incidence in an urban neonatal intensive care unit: an intervention study with before and after comparison. Int J Nurs Stud 2010;47:1245-52.

22. Jha V, Winterbottom A, Symons J, et al. Patient-led training on patient safety: A pilot study to test the feasibility and acceptability of an educational intervention. Med Teach. Published Online First: 25 March 2013.

23. Winterbottom $\mathrm{AE}$, Jha $\mathrm{V}$, Melville $\mathrm{C}$, et al. $\mathrm{A}$ randomized controlled trial of patient led training in medical education: protocol. BMC Med Educ 2010;10:90. 